\title{
A Novel Pathogenic Variant (c.592_599del) in PCDH19 in a Korean Family with Epilepsy
}

\author{
You Na Park, MD', Mi-Ae Jang, MD², Soyoung Park, MD' \\ 'Department of Pediatrics, Soonchunhyang University Bucheon Hospital, Soonchunhyang University College of Medicine, Bucheon, Korea \\ ${ }^{2}$ Department of Laboratory Medicine and Genetics, Soonchunhyang University Bucheon Hospital, Soonchunhyang University College of \\ Medicine, Bucheon, Korea
}

Received: October 19, 2019

Revised: November 11, 2019

Accepted: November 15, 2019

Corresponding authors:

Soyoung Park, MD

Department of Pediatrics,

Soonchunhyang University

Bucheon Hospital, Soonchunhyang

University College of Medicine, 170

Jomaru-ro, Wonmi-gu, Bucheon

14584, Korea

Tel: $+82-32-621-5403$

Fax: +82-32-621-5018

E-mail: hclove72@schmc.ac.kr

\section{Mi-Ae Jang, MD}

Department of Laboratory Medicine and Genetics, Soonchunhyang University Bucheon Hospital, Soonchunhyang University College of Medicine, 170 Jomaru-ro, Wonmi-gu, Bucheon 14584, Korea

Tel: +82-32-621-6275

Fax: +82-32-621-5944

E-mail: miaeyaho@schmc.ac.kr
Protocadherin 19 (PCDH19)-related epilepsy is an X-linked epilepsy syndrome characterized by afebrile or febrile seizures, starting in the early years of life, and developmental delay of variable severity [1]. It is caused by mutations in PCDH19 encoding PCDH19, a protein highly expressed during brain development, that is responsible for calcium-dependent cell-to-cell interaction. The disease is of very characteristic heredity; although it is an $\mathrm{X}$ chromosome linked disorder, it rarely affects male individuals; this is attributed to "cellular interference," where the disease could be caused by cell-to-cell signaling between mutated and unmutated cells resulting in hemizygous men being unaffected. Here, we report the case of two Korean female siblings, in which a novel pathogenic variant of $P C D H 19$, inherited from the father, caused different phenotypic expression.

$$
\text { A 23-month-old girl (Sibling 1, III:3) (Fig. 1) }
$$
was brought to our emergency room after three episodes of generalized tonic clonic seizures with low-grade fever. She had been previously healthy, except for two episodes of simple febrile seizures at 11 and 20 months old, and she had normally reached the developmental milestones without autistic features. She experienced five more seizures on the day of admission, and six times more the next day, even in the absence of fever.
The seizures varied in semiology, from generalized tonic clonic seizures lasting 3 to 5 minutes to brief unilateral tonic seizures lasting approximately 1 minute. Unresponsiveness with eye blinking lasting less than 1 minute was also detected. Her brain magnetic resonance imaging was normal, and there was no evidence of central nervous system infection in her cerebrospinal fluid study. She was neurologically intact without deficits and remained alert during the interictal periods, with no more seizures detected after levetiracetam infusion $(30 \mathrm{mg} / \mathrm{kg})$. We were informed that she had relevant family history; her 10-year-old sister had epilepsy and her mother had febrile seizures at an early age (Fig. 1). Her sister (Sibling 2, III:2) (Fig. 1) started experiencing febrile seizures at 13 months of age and had clustered brief seizures with or without fever similar to Sibling 1. Administration of multiple antiepileptic drugs was attempted for several years and the frequency of seizures decreased with age. In recent years, she had remained seizure free with levetiracetam monotherapy and her intelligence and social function were within the normal range, but her language skills were relatively delayed. Her mother had simple febrile seizures at an early age, which dissipated at 3 years of age and her intelligence was within the

Copyright(C) 2019 Korean Child Neurology Society

This is an Open Access article distributed under the terms of the Creative Commons Attribution Non-Commercial License (http://creativecommons.org/licenses/by-nc/4.0/) which permits unrestricted non-commercial use, distribution, and reproduction in any medium, provided the original work is properly cited. 
normal range.

The multitude of genes associated with epilepsy and the similarity among clinical phenotypes may complicate diagnosis; hence, we performed whole exome sequencing (WES) with the

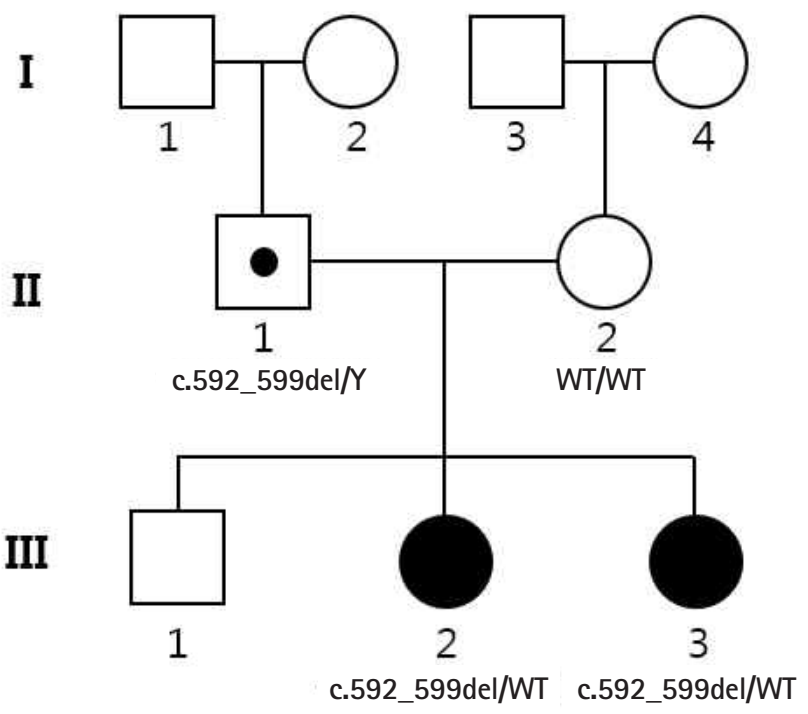

Fig. 1. Pedigree of the family, with two related cases of PCDH19related infantile epileptic encephalopathy. A pathogenic $\mathrm{PCDH} 19$ variant, c.592_599del is found hemizygous in the father (II:1) and heterozygous in the two siblings (III:2 and III:3). The mother (II:2) is homozygous for the wild-type (WT) allele. The black symbols indicate the affected individuals. informed consent of the family members (II:1, II:2, III:2, and III:3). Family-based WES is a very powerful tool for identifying pathogenic variants using expected inheritance patterns.

After screening of epilepsy-related genes, we identified a novel deletion variant (NM_001184880.1:c.592_599del) in PCDH19, which was predicted to result in frameshift and premature termination of the PCDH1 protein (p.Arg198Alafs*25) (Fig. 2). The variant was identified as a heterozygote in the two siblings (III:2 and III:3) and as a hemizygote in the father (II:1), but not in the mother (II:2). No other strong candidate genes were located in the WES results. Sibling 1 has remained seizure free for over 6 months with levetiracetam monotherapy (30 mg/kg/day) and has so far normally reached the developmental milestones.

Seizures usually start before 1 year of age, and the presence of fever-sensitive seizures may be clinically similar to the presentation of Dravet syndrome, but seizures in Dravet syndrome usually last longer than 15 minutes ( $72 \%$ of patients) and less than 5 minutes in PCDH19-related epilepsy [1]. Dravet syndrome usually involves clonic or hemiclonic-type seizures, whereas PCDH19-related epilepsy usually involves the focal or hypomotor type. Most importantly, there is a significant difference in prognosis, with remission in adolescence with variable severity of developmental delay being common in PCDH19-related epilepsy, as in our case and severe epileptic encephalopathy often seen in Dravet syndrome [2]. Regarding anticonvulsant treatment, phenytoin is re-

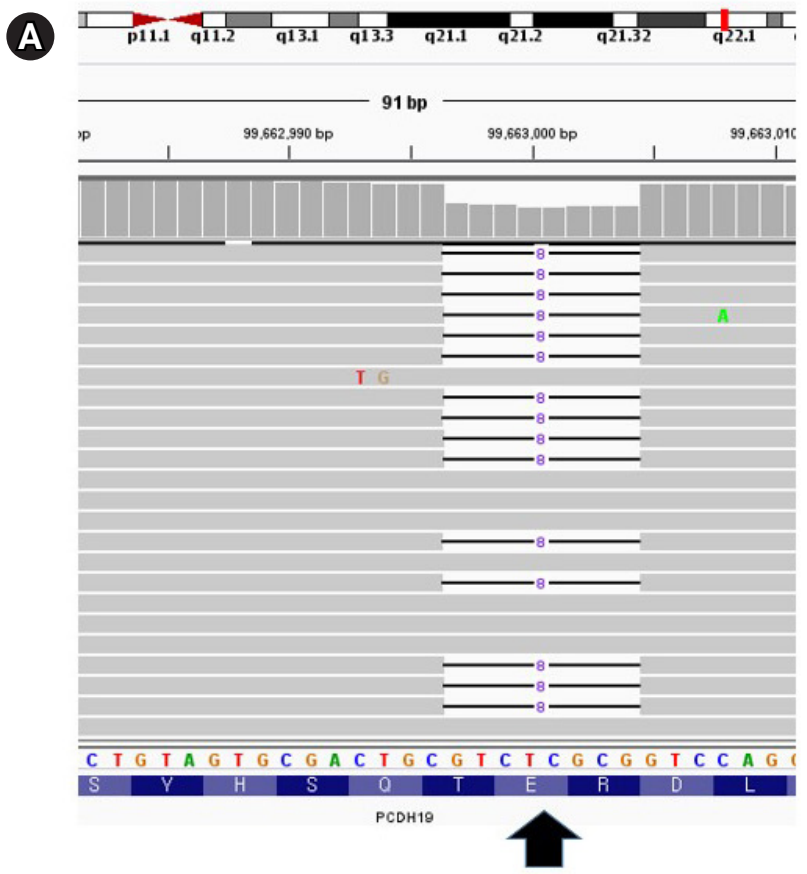

B
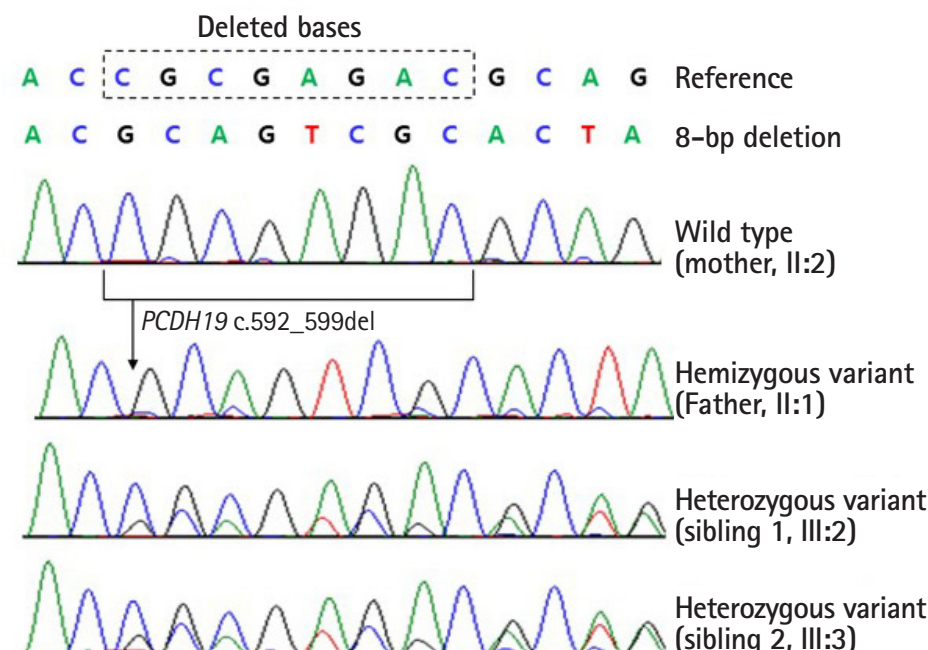

Fig. 2. Molecular analyses of PCDH19 in two siblings with epilepsy. (A) Integrative Genomics Viewer (Broad Institute and the Regents of the University of California) snapshot of a novel PCDH19 deletion variant identified by whole exome sequencing (arrow). (B) Frameshift and premature termination of the PCDH1 protein (p.Arg198Alafs*25). 
portedly effective and carbamazepine less so [3]. There have been reports of effective levetiracetam use, including in these siblings, which should be studied further [4]. Fever-sensitive seizures and prominent seizure clustering in varied patterns with short duration ( $<5$ minutes) in girls younger than 1 year, as in our cases, can provide clues for $P C D H 19$-related epilepsy to clinicians $[1,4,5]$.

\section{Supplementary materials}

Supplementary materials related to this article can be found online at https://doi.org/10.26815/acn.2019.00199.

\section{Conflicts of interest}

No potential conflict of interest relevant to this article was reported.

\section{ORCID}

You Na Park, https:/ / orcid.org/0000-0002-0193-1304

Mi-Ae Jang, https://orcid.org/0000-0002-6558-5236

Soyoung Park, https://orcid.org/0000-0001-8656-9473

\section{Author contributions}

Conceptualization: MAJ and SP. Data curation: YNP. Formal analysis: MAJ and SP. Funding acquisition: MAJ. Methodology: MAJ and SP. Project administration: MAJ and SP. Visualization: MAJ. Writing-original draft: YNP. Writing-review \& editing: MAJ and SP.

\section{Acknowledgements}

This work was supported by Soonchunhyang University Research Fund and by the Basic Science Research Program through the National Research Foundation of Korea (NRF), funded by the Ministry of Education, Science and Technology (2017R1C1B5018081).

\section{References}

1. Smith L, Singhal N, El Achkar CM, Truglio G, Rosen Sheidley B, Sullivan J, et al. PCDH19-related epilepsy is associated with a broad neurodevelopmental spectrum. Epilepsia 2018;59:67989.

2. Trivisano M, Pietrafusa N, Ciommo Vd, Cappelletti S, Palma Ld, Terracciano A, et al. PCDH19-related epilepsy and Dravet syndrome: face-off between two early-onset epilepsies with fever sensitivity. Epilepsy Res 2016;125:32-6.

3. Higurashi N, Nakamura M, Sugai M, Ohfu M, Sakauchi M, Sugawara Y, et al. PCDH19-related female-limited epilepsy: further details regarding early clinical features and therapeutic efficacy. Epilepsy Res 2013;106:191-9.

4. Lyons S, Marnane M, Reavey E, Williams N, Costello D. PCDH19-related epilepsy: a rare but recognisable clinical syndrome in females. Pract Neurol 2017;17:314-7.

5. Depienne C, LeGuern E. PCDH19-related infantile epileptic encephalopathy: an unusual X-linked inheritance disorder. Hum Mutat 2012;33:627-34. 